\title{
A highly efficient synthesis of trisubstituted quinolines using sodium hydrogensulfate on silica gel as a reusable catalyst
}

\author{
U. V. Desai, ${ }^{a}{ }^{*}$ S. D. Mitragotri, ${ }^{a}$ T. S. Thopate, ${ }^{a}$ D. M. Pore, ${ }^{a}$ and P. P. Wadgaonkar ${ }^{b}$ \\ ${ }^{a}$ Department of Chemistry, Shivaji University, Kolhapur - 416004, India \\ ${ }^{b}$ Polymer Science and Engineering Division, National Chemical Laboratory, Pune-411008, India \\ E-mail: uprabhu_desai@rediffmail.com
}

\begin{abstract}
o-Aminoaryl ketones underwent smooth condensation with a variety of $\alpha$-methylene ketones in the presence of $\mathrm{NaHSO}_{4}$. silica gel as a solid acid catalyst to afford 1,2,3-trisubstituted quinolines in excellent yields. The reusability of the catalyst has made the protocol simple, cost-effective and environmentally benign.
\end{abstract}

Keywords: $\mathrm{NaHSO}_{4}$.Silica gel, heterogeneous catalyst, Friedlander synthesis, quinolines

\section{Introduction}

Quinolines and their derivatives are receiving increasing importance due to their wide range of biological activities as anti-malarial, anti-bacterial, anti-asthmatic, anti-hypertensive, antiinflammatory, anti-platelet activity and as tyro-kinase PDGF-RTK inhibiting activity. ${ }^{1-3}$ In addition, quinolines have also been employed in the study of bio-organic and bio-organometallic processes ${ }^{4}$. Due to such a wide range of applicability in medicinal, bioorganic, industrial as well as in the fields of synthetic organic chemistry, there has been increasing interest in the development of efficient methodologies for the synthesis of quinolines.

Friedlander quinoline synthesis is one of the most straightforward approach practiced for the synthesis of quinolines. Few recent protocols reported for the synthesis of quinolines involve the use of catalysts such as $\mathrm{SnCl}_{2},{ }^{5 \mathrm{a}} \mathrm{BiCl}_{3}{ }^{5 \mathrm{~b}}, \mathrm{I}_{2}{ }^{5 \mathrm{c}}$, montmorillonite-KSF, ${ }^{6}$ ionic liquids, ${ }^{7} \mathrm{Bi}(\mathrm{OTf})_{3},{ }^{8}$ $\mathrm{Y}(\mathrm{OTf})_{3}{ }^{9}$, silverdodecatungstophosphate $\left(\mathrm{Ag}_{3} \mathrm{POW}_{12} \mathrm{O}_{40}\right),{ }^{10}$ silica sulfuric acid $^{11 \mathrm{a}}$, sulfamic $\operatorname{acid}^{11 b}$, neodymium nitrate ${ }^{11 c}$, etc. However, these methods suffer from the serious drawbacks of harsh reaction conditions, use of expensive catalysts, longer reaction times, etc. Thus, a simple and environmentally benign protocol involving the use of inexpensive and readily available reagents is desirable.

In recent years, the use of heterogeneous catalysts has received considerable interest in various disciplines including organic synthesis. They are advantageous over their homogeneous 
counterparts due to the prime advantage that in most of the cases the catalyst can be recovered easily and reused. ${ }^{12}$ Similarly the avoidance of the solvent helps in making the process environmentally benign and economically viable. Sodium hydrogen sulfate supported on silica gel $^{13}$ has been proved to be a heterogeneous catalyst of choice of many researchers, due to its ease of preparation using readily available reagents as well as its high catalytic efficiency. It has been used earlier in $\alpha$-bromination of ketones, ${ }^{14}$ in the cleavage of MOM-ethers and prenyl esters $^{15 \mathrm{a}, 15 \mathrm{~b}}$, in the synthesis of dihydropyridines ${ }^{16 \mathrm{a}}$ as well as homoallylic amines ${ }^{16 \mathrm{~b}}$, etc. Our earlier experiences with the use of heterogeneous solid acid catalyst ${ }^{17}$ as well as the recent surge in the development of solvent-free and environmentally benign protocols prompted us to test the feasibility of sodium hydrogen sulfate. $\mathrm{SiO}_{2}$ for the synthesis of quinolines and we wish to report herein a highly efficient, cost effective and environmentally benign protocol for the synthesis of 2, 4, 6-trisubstituted quinolines (Scheme 1).

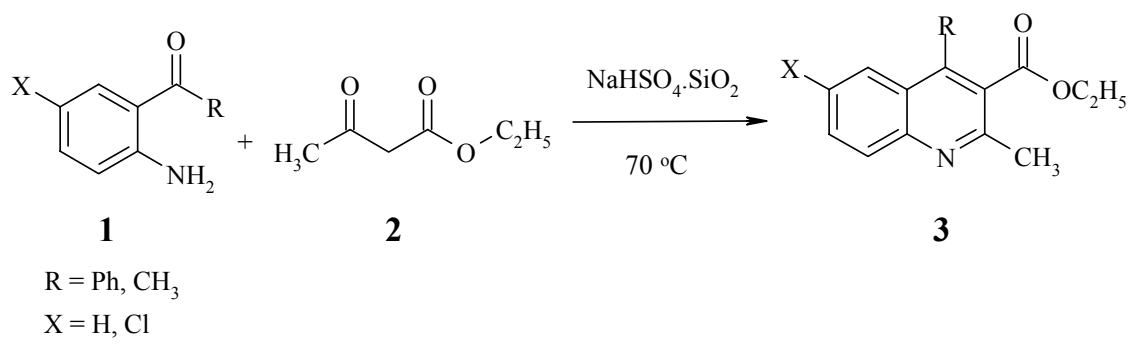

\section{Scheme 1}

\section{Results and Discussion}

In a typical reaction, a mixture of 2- aminobezophenone 1 (2 mmol), ethyl acetoacetate 2 (2.1 mmol) and $\mathrm{NaHSO}_{4} \cdot \mathrm{SiO}_{2}(200 \mathrm{mg}$ ) was stirred at room temperature (Scheme 1). The careful examination of TLC $(6 \mathrm{~h})$ showed the formation of a new product but the reaction did not go to completion even after stirring overnight. However, heating the reaction mixture for $3 \mathrm{~h}$ at $70{ }^{\circ} \mathrm{C}$ resulted in the formation of desired quinoline derivative viz. ethyl-2-methyl-4-phenyl-3quinoline carboxylate, 3a (95\%) as characterized by comparison of the mp and spectral data with that reported in the literature. ${ }^{8}$

The studies on the optimization of the reaction conditions with respect to the amount of catalyst used as well as temperature revealed that, the desired quinoline 3a couldn't be obtained in the same yield $(95 \%)$ at the temperature lower than $70{ }^{\circ} \mathrm{C}$. Furthermore, the use of common organic solvents like $\mathrm{CHCl}_{3}, \mathrm{CH}_{3} \mathrm{CN}$ or $\mathrm{EtOH}$ was not found to have any influence on the yield of $3 \mathbf{a}$ and the best results were obtained under solvent-free conditions.

The synthetic utility of the reaction under the optimized reaction conditions was evaluated by performing the reactions between 2-aminobezophenone as well as 2-amino-5chlorobenzophenone with a variety of ketones like cyclohexanone, cyclopentanone, cyclohexan- 
1,3-dione, dimedone as well as acetyl acetone. In all the cases, the corresponding quinoline derivatives were obtained in acceptable yield and purity. However, the reaction of 2aminoacetophenone with various ketones proved to be sluggish as compared to that with 2aminobenzophenone. The results of all these studies are summarized in Table 1.

Finally, the efficacy of the $\mathrm{NaHSO}_{4} \cdot \mathrm{SiO}_{2}$ as a catalyst for the synthesis of $\mathbf{3 a}$ as a model compound, was compared with that of other reported catalysts such as $\mathrm{Ag}_{3} \mathrm{PW}_{12} \mathrm{O}_{40}$, sulfamic acid, Bi (OTf $)_{3}, \mathrm{Y}(\mathrm{OTf})_{3}$ and $\mathrm{KSF}-\mathrm{Clay}$ (Table 2). It revealed that $\mathrm{NaHSO}_{4} \cdot \mathrm{SiO}_{2}$ is an equally efficient, cost-effective and environmentally benign catalyst useful in the synthesis of quinoline derivatives. From the practical point of view, the catalyst is easier to prepare from the readily available reagents and except for the isolation step wherein the solvent was essential, the reaction proceeded under solvent-free conditions. Furthermore, like other heterogeneous catalysts, it can also be reused. For example, the reaction of 2-amino-5-chlorobenzophenone and ethyl acetoacetate afforded corresponding quinoline derivative in 92, 90, and $86 \%$ yields over three successive runs.

In conclusion, we have described a highly efficient, cost-effective and environmentally benign protocol for the synthesis of 2,4,6-trisubstituted quinolines using $\mathrm{NaHSO}_{4} \cdot \mathrm{SiO}_{2}$ as a heterogeneous and reusable catalyst. This operationally simple procedure can be used as a useful alternative to the existing protocols for the synthesis of trisubstituted quinolines.

\section{Experimental Section}

General procedure. A mixture of o-amino ketone $(2 \mathrm{mmol})$, ethyl acetoacetate / acetylacetone $(2.1 \mathrm{mmol})$ and $\mathrm{NaHSO}_{4} \cdot \mathrm{SiO}_{2}(200 \mathrm{mg}, 0.52 \mathrm{mmol})$ was heated with stirring at $70{ }^{\circ} \mathrm{C}$ until completion of reaction (TLC). The reaction mixture was diluted with chloroform, the catalyst was filtered and washed with chloroform. From the combined filtrate solvent was removed under vacuum and the residue was filtered through a short column of silica gel to afford pure quinoline derivative. 
Table1. $\mathrm{NaHSO}_{4}$. $\mathrm{SiO}_{2}$-catalyzed synthesis of quinolines

\begin{tabular}{|c|c|c|c|c|c|c|}
\hline Entry & $\begin{array}{l}\text { 2-Amino- } \\
\text { ketone (1) }\end{array}$ & $\begin{array}{l}\text { Ketone } \\
(2)\end{array}$ & $\begin{array}{l}\text { Quinoline } \\
\text { (3) }\end{array}$ & $\begin{array}{l}\text { Time } \\
\text { (h) }\end{array}$ & $\begin{array}{l}\text { Yield } \\
(\%)^{a, b}\end{array}$ & $\begin{array}{l}\mathrm{Mp}\left({ }^{\circ} \mathrm{C}\right) \\
\text { Obs ( Lit })^{\text {ref }}\end{array}$ \\
\hline $\mathrm{a}$ & & & & 3 & 95 & $96(98)^{7}$ \\
\hline $\mathrm{b}$ & & & & 3 & 90 & $113(115)^{7}$ \\
\hline $\mathrm{c}$ & & & & 2.5 & 90 & $139(140-142)^{7}$ \\
\hline $\mathrm{d}$ & & & & 2 & 95 & $132(132)^{7}$ \\
\hline e & & & & 4 & 60 & $158(156-157)^{7}$ \\
\hline $\mathrm{f}$ & & & & 6 & 60 & $191(190-192)^{7}$ \\
\hline $\mathrm{g}$ & & & & 3 & 96 & $112(110)^{9}$ \\
\hline $\mathrm{h}$ & & & & 8 & 90 & $152(150)^{7}$ \\
\hline $\mathrm{i}$ & & & & 2.5 & 95 & $162(162)^{9}$ \\
\hline $\mathrm{j}$ & & & & 3 & 85 & $96(95)^{9}$ \\
\hline
\end{tabular}


$\mathrm{k}$<smiles>CC(=O)c1ccccc1N</smiles>

1

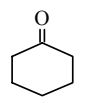

$\mathrm{H}_{3} \mathrm{C}$

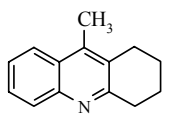

4

4

65

${ }^{\mathrm{a}}$ Yields refer to pure isolated products; ${ }^{\mathrm{b}} \mathrm{All}$ compounds gave satisfactory spectroscopic analysis

Table 2. Comparison of the efficiency of $\mathrm{NaHSO}_{4} \cdot \mathrm{SiO}_{2}$ for synthesis of $\mathbf{3 a}$

\begin{tabular}{lllll}
\hline Entry & Catalyst & $\begin{array}{l}\text { Time } \\
(\mathrm{h})\end{array}$ & $\begin{array}{l}\text { Yield } \\
(\%)\end{array}$ & Ref. \\
\hline 1 & $\mathrm{KSF}-C l a y$ & 6 & 75 & {$[6]$} \\
2 & $\mathrm{Ag}_{3} \mathrm{PW}_{12} \mathrm{O}_{40}$ & 3 & 92 & {$[10]$} \\
3 & $\mathrm{Sulfamic}$ acid & 0.5 & 92 & {$[11]$} \\
4 & $\mathrm{Bi}(\mathrm{OTf})_{3}$ & 3.5 & 86 & {$[8]$} \\
5 & $\mathrm{Y}_{3}(\mathrm{OTf})_{3}$ & 4 & 89 & {$[9]$} \\
6 & $\mathrm{NaHSO}_{4}$.silicagel & 3 & 95 & -- \\
\hline
\end{tabular}

\section{Spectral Data}

Ethyl-2-methyl-4-phenylquinoline-3-carboxylate (3a). IR (KBr): 3060, 2979, 930, 1730, 1579, 1488, 1400, 1298, 1230, 1068, $770 \mathrm{~cm}^{-1} ;{ }^{1} \mathrm{H}$ NMR (400 MHz, $\left.\mathrm{CDCl}_{3}\right): \delta=0.97(\mathrm{t}, J=7$ $\mathrm{Hz}, 2 \mathrm{H}), 2.82(\mathrm{~s}, 3 \mathrm{H}), 4.12(\mathrm{q}, J=7 \mathrm{~Hz}, 2 \mathrm{H}) 7.30-7.55(\mathrm{~m}, 7 \mathrm{H}), 7.6(\mathrm{~d}, 1 \mathrm{H}), 7.75(\mathrm{t}, 1 \mathrm{H}), 8.15(\mathrm{~d}, J$ $=7 \mathrm{~Hz}, 1 \mathrm{H})$; EIMS: $\mathrm{m} / \mathrm{z}=291\left(\mathrm{M}^{+}\right)$.

1-(2-Methyl-4-phenylquinoline-3-yl)ethanone (3b). IR (KBr): 3061, 2960, 1700, 1567, 1441, 1396, $1215,766,704 \mathrm{~cm}^{-1} ;{ }^{1} \mathrm{H}$ NMR: $\delta=2(\mathrm{~s}, 3 \mathrm{H}), 2.7(\mathrm{~s}, 3 \mathrm{H}), 7.2-7.5(\mathrm{~m}, 6 \mathrm{H}), 7.6 \quad$ (d, $J=8 \mathrm{~Hz}, 1 \mathrm{H}), 7.7(\mathrm{t}, J=7 \mathrm{~Hz}, 1 \mathrm{H}), 8.1(\mathrm{~d}, J=8 \mathrm{~Hz}, 1 \mathrm{H})$; EIMS: $\mathrm{m} / \mathrm{z}=261\left(\mathrm{M}^{+}\right)$.

9- Phenyl-1,2,3,4- tertrahydroacridine (3c). IR (KBr): 3061, 2936 , 2860 , 1572 , 1488 , 1440, $1220,768,701 \mathrm{~cm}^{-1} ;{ }^{1} \mathrm{H}$ NMR : $\delta=1.78-1.85(\mathrm{~m}, 2 \mathrm{H}), 1.9-2.05(\mathrm{~m}, 2 \mathrm{H}), 2.6(\mathrm{t}, J=7 \mathrm{~Hz}$, $2 \mathrm{H}), 3.2(\mathrm{t}, J=7 \mathrm{~Hz}, 2 \mathrm{H}), 7.2-7.25(\mathrm{~m}, 4 \mathrm{H}), 7.45-7.65(\mathrm{~m}, 4 \mathrm{H}), 8.05(\mathrm{~d}, J=8 \mathrm{~Hz}, 1 \mathrm{H})$; EIMS: $\mathrm{m} / \mathrm{z}=259\left(\mathrm{M}^{+}\right)$.

9-Phenyl-2,3-dihydro-1-H-cyclopenta(b) quinoline (3d). IR (KBr): 2928, 1569, 1478, 1348, 1164, 1076, 939, 832, 819, 775, 776, 708, $618 \mathrm{~cm}^{-1} ;{ }^{1} \mathrm{H}$ NMR : $\delta=1.98(\mathrm{~m}, 2 \mathrm{H}), 2.9(\mathrm{t}, J=8$ $\mathrm{Hz}, 2 \mathrm{H}), 3.23$ ( t $, J=8 \mathrm{~Hz}, 2 \mathrm{H}), 7.35-7.65(\mathrm{~m}, 9 \mathrm{H}), 8.15(\mathrm{~d}, J=8 \mathrm{~Hz}, 1 \mathrm{H}) ; \mathrm{EIMS}: \mathrm{m} / \mathrm{z}=245$ ( $\left.\mathrm{M}^{+}\right)$.

9-Phenyl-1, 2,3,4-tetrahydro-1-acridinone (3e). IR (KBr): 2948, 1691, 1552, 1485, 1392, 1220, 1161, 1023, 771, 757, 706, $539 \mathrm{~cm}^{-1}$; ${ }^{1} \mathrm{H}$ NMR: $\delta=2.25$ (quin, $2 \mathrm{H}$ ), 2.70 (t, $J=8 \mathrm{~Hz}, 2 \mathrm{H}$ ), $3.40(\mathrm{t}, J=8 \mathrm{~Hz}, 2 \mathrm{H}), 7.2(\mathrm{~m}, 2 \mathrm{H}), 7.35-7.6(\mathrm{~m}, 5 \mathrm{H}), 7.8(\mathrm{~m}, 1 \mathrm{H}), 8.1(\mathrm{~d}, 1 \mathrm{H}) ; \mathrm{EIMS}: \mathrm{m} / \mathrm{z}=$ $273\left(\mathrm{M}^{+}\right)$.

3,3-Dimethyl-9-phenyl-1, 2,3,4 tetrahydro-1-acridinone (3f). IR(KBr): 3061, 2956, 1712, 
1602, 1573, 1206, 735 $\mathrm{cm}^{-1} ;{ }^{1} \mathrm{H}$ NMR: $\delta=1.1(\mathrm{~s}, 6 \mathrm{H}), 2.59(\mathrm{~s}, 2 \mathrm{H}), 3.3(\mathrm{~s}, 2 \mathrm{H}), 7.1-7.2(\mathrm{~m}, 2 \mathrm{H})$, 7.3-7.55 (m, 5H), $7.78(\mathrm{t}, 1 \mathrm{H}), 8.1(\mathrm{~d}, J=8 \mathrm{~Hz}, 1 \mathrm{H})$; EIMS: $(\mathrm{m} / \mathrm{z})=301\left(\mathrm{M}^{+}\right)$.

Ethyl-6- chloro - 2- methyl-4- phenylquinoline- 3-carboxylate. (3g). IR (KBr): 3066, 2989, 1727, 1605, 1223, 906, $733 \mathrm{~cm}^{-1}$; ${ }^{1} \mathrm{H}$ NMR: $\delta=0.90(\mathrm{t}, J=7 \mathrm{~Hz}, 3 \mathrm{H}), 2.75(\mathrm{~s}, 3 \mathrm{H}), 3.95(\mathrm{q}, J$ $=7 \mathrm{~Hz}, 2 \mathrm{H}), 7.25-7.35(\mathrm{~m}, 2 \mathrm{H}), 7.4-7.5(\mathrm{~m}, 4 \mathrm{H}), 7.6(\mathrm{dd}, J=8 \mathrm{~Hz}, 1 \mathrm{H}), 7.95(\mathrm{~d}, J=8 \mathrm{~Hz}, 1 \mathrm{H})$; EIMS: $\mathrm{m} / \mathrm{z}=325\left(\mathrm{M}^{+}\right)$.

1-(6-Chloro-2-methyl-4-phenylquinoline) ethanone (3h). IR (KBr): 3026, 2950, 1695, 1605, $692 \mathrm{~cm}^{-1} ;{ }^{1} \mathrm{H}$ NMR : $\delta=2.0(\mathrm{~s}, 3 \mathrm{H}), 2.7(\mathrm{~s}, 3 \mathrm{H}), 7.25-7.40(\mathrm{~m}, 2 \mathrm{H}), 7.5-7.6(\mathrm{~m}, 3 \mathrm{H}), 7.7(\mathrm{~m}$, $2 \mathrm{H}), 8.0(\mathrm{~d}, J=8 \mathrm{~Hz}, 1 \mathrm{H})$; EIMS: $\mathrm{m} / \mathrm{z}=295\left(\mathrm{M}^{+}\right)$.

7- Chloro-9-phenyl-1,2,3,4-tetrahydroacridine (3i). IR (KBr): 3060, 2945, 1602, 1571, 1482, 1215, $705 \mathrm{~cm}^{-1} ;{ }^{1} \mathrm{H}$ NMR : $\delta=1.65-1.75(\mathrm{~m}, 4 \mathrm{H}), 1.80-1.95 \quad(\mathrm{~m}, 2 \mathrm{H}), 2.55(\mathrm{t}, J=8 \mathrm{~Hz}, 2 \mathrm{H})$, $3.1(\mathrm{t}, J=8 \mathrm{~Hz}, 2 \mathrm{H}), 7.1-7.25(\mathrm{~m}, 3 \mathrm{H}), 7.35-7.55(\mathrm{~m}, 4 \mathrm{H}), 7.9(\mathrm{~d}, J=8 \mathrm{~Hz} 1 \mathrm{H}) ; \mathrm{EIMS}: \mathrm{m} / \mathrm{z}=$ $293\left(\mathrm{M}^{+}\right)$.

7-Chloro-9-phenyl-2,3-dihydro-1-H-cyclopenta (b) quinoline (3j). IR ( $\mathrm{KBr}$ ): 3060, 2956, 1605, 1484, 828, $715 \mathrm{~cm}^{-1}$; ${ }^{1} \mathrm{H}$ NMR: $\delta=2.2$ (quin, 2H), $2.90(\mathrm{t}, J=8 \mathrm{~Hz}, 2 \mathrm{H}), 3.25(\mathrm{t}, J=8$ $\mathrm{Hz}, 2 \mathrm{H}), 7.30-7.40(\mathrm{~m}, 2 \mathrm{H}), 7.45-7.60(\mathrm{~m}, 5 \mathrm{H}), 8.0$ (d, J=8 Hz, 1H); EIMS: m/z = $279\left(\mathrm{M}^{+}\right)$.

Ethyl-2,4-dimethylquinoline-3-carboxylate (3k). IR (Neat): 3066, 2931, 1870, 1724, 1616, 1587, 1212, 1082, $758 \mathrm{~cm}^{-1} ;{ }^{1} \mathrm{H}$ NMR : $\delta=1.42(\mathrm{t}, J=8 \mathrm{~Hz} 3 \mathrm{H}), 2.65(\mathrm{~s}, 3 \mathrm{H}), 2.75(\mathrm{~s}, 3 \mathrm{H})$, $4.5(\mathrm{q}, J=8 \mathrm{~Hz}, 2 \mathrm{H}), 7.45(\mathrm{~m}, 1 \mathrm{H}), 7.68(\mathrm{~m}, 1 \mathrm{H}), 8.0(\mathrm{~m}, 2 \mathrm{H})$.

MS: $\mathrm{m} / \mathrm{z}(\%)=229(91), 214(8), 200(10), 183(100), 156(50), 128(20), 115(37), 89(18), 77(10)$; Calcd for $\mathrm{C}_{14} \mathrm{H}_{15} \mathrm{O}_{2} \mathrm{~N}$ : C,73.34; H, $6.59 ; \mathrm{N}, 6.11$. Found : C,73.14; H, $6.38 ; \mathrm{N}, 6.03$.

9-Methyl- 1,2,3,4- tertrahydroacridine (3I). IR (KBr) 2928, 1569, 1478, 1348, 1164, 1076, 939, 832, 819, 775, 752, 708, $618 \mathrm{~cm}^{-1}$; ${ }^{1} \mathrm{H}$ NMR (400 MHz, CDCl3): $\delta=1.8-1.85(\mathrm{~m} \mathrm{2H}), 2.2$ $(\mathrm{s} 3 \mathrm{H}), 2.6(\mathrm{~m}, 2 \mathrm{H}), 2.8(\mathrm{~m}, 2 \mathrm{H}), 3.1(\mathrm{~m}, 2 \mathrm{H}), 7.5(\mathrm{~m}, 1 \mathrm{H}), 7.6(\mathrm{~m}, 1 \mathrm{H}), 8(\mathrm{~d} J=8 \mathrm{~Hz} 1 \mathrm{H})$, $8.1(\mathrm{~d} J=8 \mathrm{~Hz} 1 \mathrm{H})$.

\section{Acknowledgements}

One of the authors (UVD) wishes to thank UGC, New Delhi for financial assistance [F.3072/2004(SR)], TST thanks UGC authorities for the award of Teacher Fellowship and SDM thanks UGC authorities for Junior Research Fellowship.

\section{References}

1. Larsen, R. D. ; Corley, E. G.; King, A. O. ; Carrol. J. D.; Davis, P.; Verhoeven, T. R; Reider, P. J. ; Lablle, M. ; Gauthier, J. Y. ; Xiang, Y. B.; Zamboni, R. J. J. Org. Chem. 1996, 61, 3398. (b) Chen, Y. L.; Fang, K. C.; Sheu, J. Y.; Hsu, S. L.; Tzeng, C. C. J. Med. Chem. 2001, 44, 2374. (c) Roma, G.; Braccio, M. D.; Gmattioli, G. F.; Ghia, M. Eur. J. Med. Chem. 
2000, 35, 1021.

2. Kalluraya, B.; Serrnivasa, S.; Farmaco 1998, 53, 399. (b) Doube, D.; Blouin, M.; Brideau, C.; Chan, C.; Desmarais, S.; Eithier, D.; Fagueyret, J. P; Friesen, R. W.; Girard, M.; Girard, Y.; Guay, J.; Tagari, P.; Young, R. N., Bioorg. Med. Chem. Lett. 1998, 8, 1255.

3. Ko, T. C.; Hour, M. J.; Liem, J. C.; Teng,C. -M.; Lee, K -H.; Kuo, S.-C.; Huang, L.-J.; Bioorg. Med. Chem. Lett. 2001, 11, 279. (b) Ferrarini, P. L.; Mori, C.; Badwneh, M.; Manera, C.;Martinelli, A.; Miceli, M.; Ramagnoli, F.; Saccomanni, G. J. Hetetocycl. Chem. 1997, 34, 1501. (c) Maguire, M. P.; Sheets, K. R.; Mcverty, K.; Spada, A. P.; Zilberstain, A. J. Med. Chem. 1994, 37, 2129.

4. Saito, I.; Sando, S.; Nakatani, K. Bio. Org. Med. Chem. 2001, 9, 2381.

5. (a)Arumugan, P.; Karthikeyn, G.; Atchudan, R.; Murlidharan, D.; Perumal. P. T. Chem. Lett. 2005, 34, 314. (b) Jia,C.-S ; Wang, G.-W. Tetrahedron Lett. 2006, 47, 289. (c) Wu, J.; Xia, H.-G. ; Gao, K. Org.Biomol.Chem. 2006, 4, 126.

6. Yadav, J. S.; Subba Reddy, B. V.; Sunitha, V.; Srinivasa Reddy K.; Ramakrishna, K. V. S. Tetrahedron Lett. 2004, 45, 7947.

7. Palimkar, S. A.; Siddiqui, S. A.; Daniel, T.; Lahoti, R. J.; Srinivasan, K. V. J. Org. Chem. 2003, 68, 9371.

8. Yadav, J. S.; Reddy, B. V. S.; Premalatha, K. Synlett 2004, 963.

9. De, S. K.; Gibbs, R. A. Tetrahedron Lett. 2005, 46, 1647.

10. Yadav, J. S.; Reddy, B. V. S.; Sreedhar, P.; Srinivasa Rao, R.; Nagaiah, K. Synthesis 2004, 2381.

11. (a).Shaabani ,A.; Soleimani, E.; Badri, Z. Montasch fur Chem. 2006,137,181. (b)Yadav, J. S.; Purushottam Rao, P.; Sreenu, D.; Srinivasa Rao, R.; Naveen Kumar, V.; Nagaiah, K.; Prasad, A. R. Tetrahedron Lett. 2005, 46, 7249. (c) Varala, R.; Enugala, R. ; Adapa, S.R. Synthesis 2006, 3825.

12. Clark, J. H. Acc. Chem. Res. 2002, 35, 791.

13. Breton, G. W. J. Org Chem. 1997, 62, 8952.

14. Das, B.; Venkateswarlu, K.; Mahender, G.; Mahender, I. Tetrahedron Lett. 2005, 46, 3041.

15. (a) Ramesh, C.; Ravindrnath, N.; Das, B. J. Org. Chem. 2003, 68, 7101. (b) Ramesh, C.; Mahender, G.; Ravindrnath, N.; Das, B. Tetrahedron Lett. 2003, 44, 1465.

16. Chari, M. A.; Syamasundar, K. Cat. Commun. 2005, 6, 624. (b) Das, B.; Ravikanth, B.; Laxminarayana, K.; Rao, B. V. J. Mol. Cat. A: Chemical 2006, 253, 92.

17. (a) Desai, U. V.; Thopate, T. S.; Pore, D. M.; Wadgaonkar, P. P. Cat. Commun., 2006, 7, 506. (b) Desai, U. V.; Pore, D. M.; Mane, R B.; Wadgaonkar, P. P. Synth Commun, 2004, 34, 2135. (c) Desai, U. V.; Thopate, T. S.; Pore, D. M.; Wadgaonkar, P. P. Ind. J. Chem. Sec-B, 2006, 45B, 1291. (d) Pore, D. M.; Desai, U. V.; Thopate, T. S.; Wadgaonkar, P. P. Arkivoc, 2006, xii, 75 